\title{
Correction to: Extranodal lymphoma arising within the maxillary alveolus: a systematic review
}

\author{
David MacDonald ${ }^{1} \cdot$ Sean Lim ${ }^{1}$
}

Published online: 14 July 2018

๑) Japanese Society for Oral and Maxillofacial Radiology and Springer Nature Singapore Pte Ltd. 2018

\section{Correction to: Oral Radiology (2018) 34:185 https://doi.org/10.1007/s1 1282-017-0309-5}

Owing to an unfortunate mistake at Springer's end, the original article was published with errors which are corrected with this erratum as suggested by the author. The authors are not responsible for this procedural lapse.

In the original publication of the article, in "Results", the sentence that reads as "Unlike Tables 1 and 2 reports non-comments ... mobility at presentation as "No"." should read as "Unlike Table 1, Table 2 recorded non-comments ... mobility at presentation as "No".".

Under the heading "Discussion", in ninth paragraph, the sentence that reads as "More than half ... cause numbness." should read as "More than half of the 13 lymphomas in Table 2 measured by advanced imaging modalities were of substantial dimensions, and although some can be expected to have reached the inferior orbital nerve, this may not be enough to cause numbness.".

The original article has also been updated.

Errors have subsequently been identified in the original publication, and the following correction should be noted:

The incorrect copyright line in Springer link is changed in this correction.

The original article can be found online at https://doi.org/10.1007/ s11282-017-0309-5.

David MacDonald

dmacdon@dentistry.ubc.ca

1 Division of Oral and Maxillofacial Radiology, Faculty of Dentistry, UBC, 2199 Wesbrook Mall, Vancouver, BC V6T 1Z3, Canada 